\title{
Application of CAD-CAM Milled Zirconia Attachment in Kennedy's Class III Partially Dentate Situation: A Series of Clinical Reports
}

\author{
Narendra Basutkar ${ }^{1}$, Afnan AlJohani ${ }^{2}$, Mohammad AlHindi ${ }^{3}$
}

\begin{abstract}
Background: Rapid developments of ceramic technology have increased applications of zirconia in dentistry. It is widely used for full, partial coverage crowns, inlays, onlays, post, and core. Though recommended, zirconia has not been widely used as precision attachment to support a cast metal partial denture prosthesis.

Aim: The purpose of this clinical report was to use CAD-CAM milled zirconia as an extra coronal attachment to retain a cast metal partial denture prosthesis.

Case description: Two partially dentate patient requiring removable prosthesis and anterior fixed dental prosthesis were considered for the study. One piece CAD-CAM milled anterior zirconia bridge with extra-coronal attachment at distal ends was fabricated and metal removable prosthesis was delivered. Patients were followed up periodically for two years. Two year periodic follow up showed application of CAD-CAM milled zirconia attachments to retain metal removable prosthesis was clinically acceptable. Patient reported satisfaction with function and esthetics. Conclusion: Use of CAD-CAM milled zirconia anterior bridge with extracoronal attachment to retain metal removable prosthesis can enhance esthetics with increased patient acceptance.

Keywords: Attachments, CAD-CAM, Zirconia.

International Journal of Prosthodontics and Restorative Dentistry (2019): 10.5005/jp-journals-10019-1235
\end{abstract}

\section{INTRODUCTION}

Zirconia (also referred to as "ceramic steel") has excellent mechanical properties. Flexural strength measuring up to 900-1200 MPa and Hardness up to 1200 HV have been reported. ${ }^{1}$ These values are same as metals used in metal ceramic restorations and significantly higher than all other ceramic materials used in dentistry. Over the last two decades, zirconia technology has propelled a rapid development of metal-free dentistry, which may provide high biocompatibility, enhanced esthetics, and improved material strength. ${ }^{2,3}$ Apart from its high flexural strength and hardness, zirconia is popular in dentistry also because of its chemically inertness, higher corrosion resistance, and extremely low thermal conductivity.

Two clinical cases has been presented here showing application of a CAD-CAM milled single unit zirconia anterior bridge with extracoronal attachment at its distal ends to retain cast metal partial denture prosthesis in two Kennedy's class III partially dentate patients.

\section{Case Description}

\section{Case 1}

A 48-year-old female patient was referred to the department of prosthdontics at the Ibn Sina National College for Medical Studies, Jeddah, Kingdom of Saudi Arabia for replacement of her missing teeth. Maxillary preoperative condition can be seen in Figure 1A. Small maxillary tuberosity can be seen. No discomfort was reported because of the tuberosity. Patient motivation to good hygiene practice was initiated. After preliminary diagnosis, preliminary treatment plan was formulated and accordingly hopeless teeth
${ }^{1-3}$ Department of Prosthodontics, Ibn Sina National College for Medical Studies, Jeddah, Kingdom of Saudi Arabia

Corresponding Author: Narendra Basutkar, Department of Prosthodontics, Ibn Sina National College for Medical Studies, Jeddah, Kingdom of Saudi Arabia, Phone: +966 557344673, e-mail: docnaren28feb@ibnsina.edu.sa

How to cite this article: Basutkar N, Aljohani A, et al. Application of CAD-CAM Milled Zirconia Attachment in Kennedy's Class III Partially Dentate Situation: A Series of Clinical Reports. Int J Prosthodont Restor Dent 2019;9(2):63-66.

Source of support: Nil

Conflict of interest: None

number 15, 14, 12, and 23 were extracted. Patient was referred to the endodontic department for root canal treatment for the teeth number 21 and 22.

Patient was presented with multiple options to the replacement of missing teeth. Dental implants were discussed and were ruled out because of financial considerations and a prolonged treatment duration.

One piece anterior fixed bridge from 13 to 22 was considered to replace missing teeth number 12 . Extracoronal attachment on distal ends of the fixed dental prosthesis (FDP) to retain the metal removable partial denture was planned. Considering the patient demands for esthetics, to eliminate the display of clasp in the anterior region and to enhance the retention of the prosthesis, a CAD-CAM milled single-piece monolithic zirconia bridge with attachments was planned. Teeth were prepared according to all ceramic preparation guidelines (Fig. 1B). Final impressions

(c) The Author(s). 2019 Open Access This article is distributed under the terms of the Creative Commons Attribution 4.0 International License (https://creativecommons. org/licenses/by-nc/4.0/), which permits unrestricted use, distribution, and non-commercial reproduction in any medium, provided you give appropriate credit to the original author(s) and the source, provide a link to the Creative Commons license, and indicate if changes were made. The Creative Commons Public Domain Dedication waiver (http://creativecommons.org/publicdomain/zero/1.0/) applies to the data made available in this article, unless otherwise stated. 


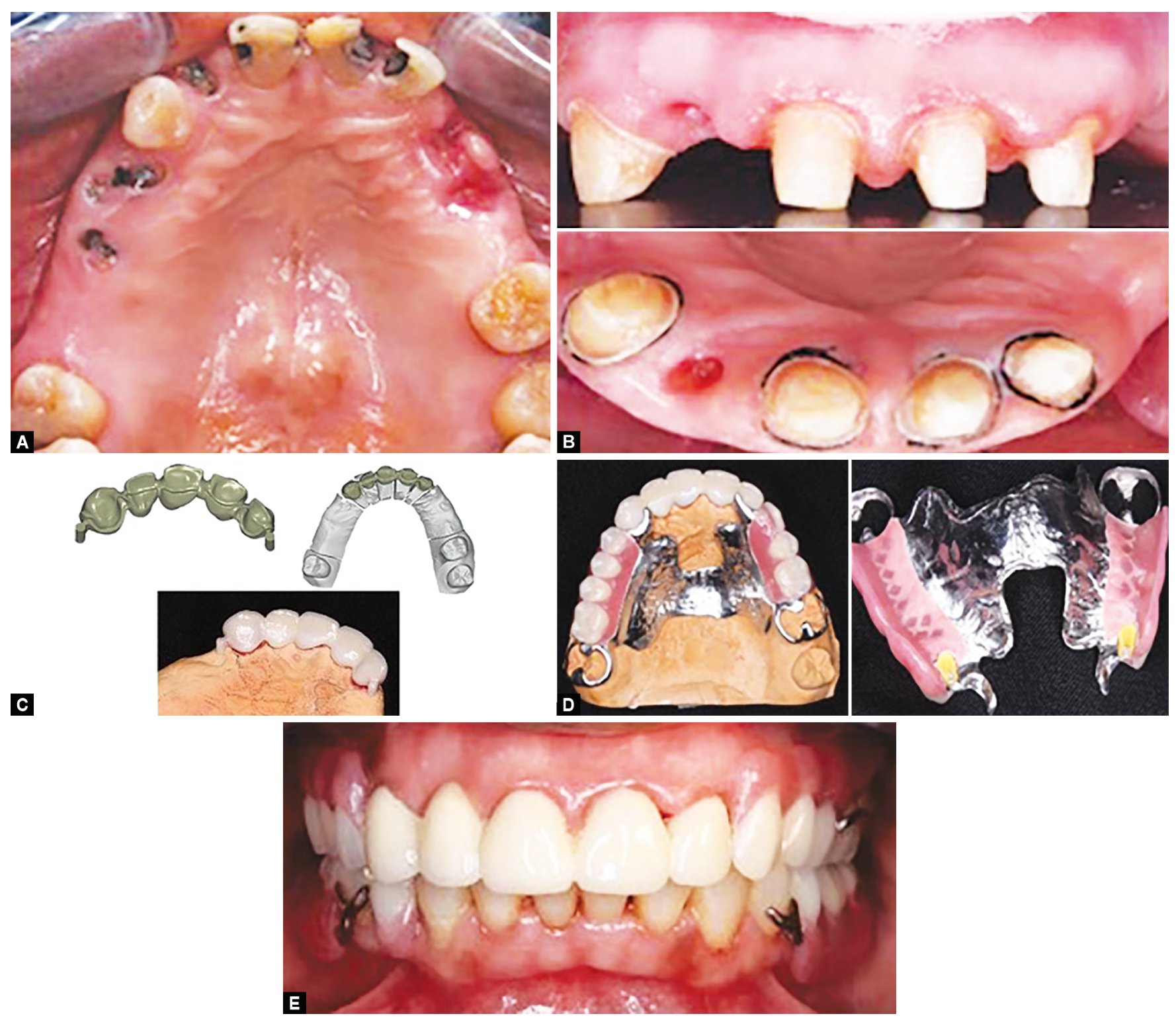

Figs $1 \mathrm{~A}$ to E: Procedural steps in Case 1

were made with additional silicone elastomeric material using a custom tray.

Computer-aided designing of the zirconia (zirCAD, Ivoclar) FDP can be seen in Figure 1C. Computer-aided milling was carried out in a partially sintered Y TZP block and final prosthesis can be seen on the cast (Fig. 1C). Extracoronal attachment was kept at $4 \mathrm{~mm}$ long and $1.5 \mathrm{~mm}$ thick to provide adequate strength. Kennedy's class III cast metal removable prosthesis was designed and fabricated (Fig. 1D). Major connector design was palatal strap anterior to the maxillary tuberosity. Zirconia bridge was cemented using resin-modified GIC and a removable partial metal prosthesis was inserted. Occlusion was adjusted for high points and uniform equal intensity contacts were established. Postinsertion instructions regarding insertion, removal of prosthesis, chewing food, and hygiene measures were given to the patient. The patient was followed at 24 hours, with 1-week interval. Patient concerns and comfort were noted (Fig. 1E).

\section{Case 2}

The second patient was female aged 39 years. Preoperative condition can be seen in Figure 2A. Patient was educated and motivated for good hygiene practice measures. A similar treatment plan involving anterior one piece zirconia bridge (zirCAD, ivoclar) with extra coronal sliding-type attachment on its distal ends and replacing missing posterior teeth with metal removable partial denture prosthesis was proposed and finalized after patient consent. Similar treatment protocol as with first case was followed, can be seen in Figures 2B to $\mathrm{E}$.

\section{Discussion}

Zirconia exists as a polymorphic ceramic material in its unalloyed state. It has three crystallographic forms: monoclinic (M) - from room temperature to $1170^{\circ} \mathrm{C}$; tetragonal $(\mathrm{T})$ - from $1170^{\circ} \mathrm{C}$ to $2370^{\circ} \mathrm{C}$; and cubic (C) - from $2370^{\circ} \mathrm{C}$ to the melting point. ${ }^{3}$ 


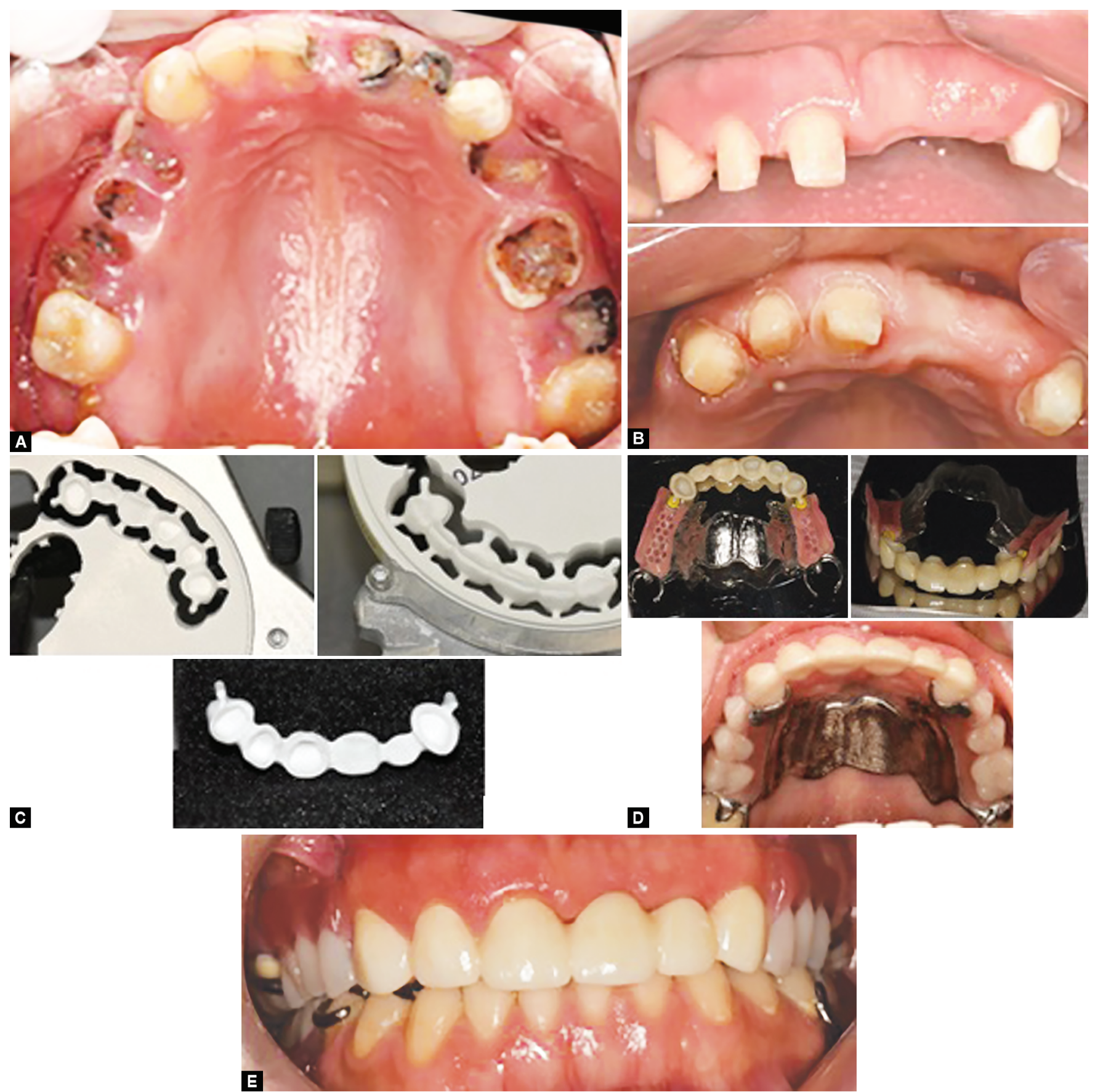

Figs 2A to E: Procedural steps in Case 2

The tetragonal phase exhibits excellent mechanical properties, enabling its various applications in dentistry. Celia $\left(\mathrm{CeO}_{2}\right)$, magnesia $(\mathrm{MgO})$, or yttria oxides $\left(\mathrm{Y}_{2} \mathrm{O}_{3}\right)$ are added to stabilize the tetragonal phase of zirconia and to prevent its transformation to the monoclinic phase. These stabilizing oxides preserve positive mechanical properties of the tetragonal phase. ${ }^{7}$

Yttrium-stabilized tetragonal zirconia particles (Y-TZP) are used in dentistry for full- and partial-coverage crowns and bridges, veneers, inlays, onlays, post and core, primary telescopic crowns, orthodontic brackets, implant, implant abutments, and extracoronal attachments. ${ }^{6}$

Laboratory studies have shown that zirconia checks all the boxes and is becoming the main choice as a restorative material for dentists across the world. ${ }^{4}$ Currently there are two different types of Y-TZP attachments available, a ball attachment for overdentures as a part of zirconia post and an extracoronal, cylindrical, or ball attachment for removable partial dentures. ${ }^{3}$ However there is no literature available regarding either clinical performance or effectiveness. In these two cases, a custom cylindrical extracoronal attachment design providing sliding fit was fabricated using CAD-CAM.

A monolithic zirconia one piece CAD-CAM fabricated anterior bridge with extra coronal attachment was considered as it will better distribute forces of mastication over the entire anterior segment. The space available for attachment was carefully evaluated during the jaw relation procedure. For a better strength, 
an optimum attachment of 1.5- to 2-mm thickness and a length of 3-4 mm was fabricated. Exo CAD system of CAD-CAM was used. High strength, biocompatibility, and obvious esthetic superiority were the reasons for the selection of zirconia as material for the restoration.

Tanner et al. have reported $95.7 \%$ of survival rate of multiunit zirconia bridges in their eight-year-old retrospective clinical study. Few of the failures reported were because of the chipping of veneered ceramic. They also reported elevated bleeding on probing around abutment teeth. This was attributed to insufficient embrasures due to thick connector areas of the zirconia framework. ${ }^{5}$

Some of the other concerns that were reported in the literature regarding the use of zirconia in dentistry is that its long-term performance is compromised by its susceptibility to hydrothermal degradation. Hydrothermal effects are generally reported between $200^{\circ} \mathrm{C}$ and $400^{\circ} \mathrm{C}$, which may not be a matter of concern for its use in dentistry. ${ }^{6}$ A long-term evaluation is needed to confirm surface disintegration, leading to microcracks due to longer exposure times at oral temperature.

Two-year followup of both cases were done. Patient did not report any problems with retention of the partial denture or any difficulty associated with zirconia attachment. Clinical examination of the zirconia CAD-CAM milled prosthesis and partial denture also revealed nothing abnormal. There were no surface cracks or surface disintegration. The gingival marginal tissue response was good and patient reported satisfaction with overall esthetics.

The future of ceramics in dentistry is promising because of increased demand for tooth coloured restorations and reduced use of amalgams and cast metals. Strong, highly biocompatible, and esthetic Y-TZP ceramic can replace traditionally used porcelain fused to the metal bridge with extra coronal attachments.

\section{Conclusion}

Clinical interest in zirconia has rapidly increased with development of newer CAD-CAM systems. Currently zirconia as dental restorative material is becoming the main choice for clinicians. Long-term clinical observations are needed to confirm this short-term observations.

\section{AcKowledgment}

The authors thank Yasmin Dental labs, Jeddah, for their support with CAD-CAM works.

\section{References}

1. Anusavice KJ, Shen C, et al. Phillip's science of dental materials. 12th ed., Elsevier Publications; 2013.

2. Vagkopoulou T, Koutayas SO, et al. Zirconia in dentistry: Part 1. Discovering the nature of an upcoming bioceramic. Eur J Esthet Dent 2009;4(2):130-151.

3. Koutayas SO, Vagkopoulou T, et al. Zirconia in dentistry part 2: Evidence based clinical breakthrough. Eur J Esthet Dent 2009;4(4):348-380.

4. Spehar D, Jakovac M. New knowledge about Zirconium-Ceramic as structural material in fixed prosthodontics. Acta Stomatol Croat 2015;49(2):137-144. DOI: 10.15644/asc49/2/7.

5. Tanner J, Niemi $\mathrm{H}$, et al. Zirconia single crowns and multiple-unit FDPs-An up to 8-year retrospective clinical study. J Dent 2018; 79:96-101. DOI: 10.1016/j.jdent.2018.10.012.

6. Cavalcanti AN, Foxton RM. Y-TZP ceramics: key concepts for clinical application. Oper Dent 2009;34(3):344-351. DOI: 10.2341/ 08-79.

7. Manicone PF, Rossi lommetti P, et al. An overview of zirconia ceramics: basic properties and clinical applications. J Dent 2007;35(11):819-826. DOI: 10.1016/j.jdent.2007.07.008. 